\title{
Exploração de recursos alimentares por psitacídeos (Aves: Psittaciformes) em uma área urbana no Brasil
}

\author{
Carolina Prudente Marques ${ }^{1}$ \\ Diogo Ferreira do Amaral ${ }^{2 *}$ \\ Vinicius Guerra ${ }^{3}$ \\ Alexandre Gabriel Franchin ${ }^{1}$ \\ Oswaldo Marçal Júnior ${ }^{1}$ \\ ${ }^{1}$ Laboratório de Ornitologia e Bioacústica, Instituto de Biologia, Universidade Federal de Uberlândia Campus Umuarama, \\ Rua Ceará, s/n, CEP 38405-315, Uberlândia - MG, Brasil \\ ${ }^{2}$ Programa de Pós-Graduação em Conservação de Recursos Naturais do Cerrado \\ Instituto Federal Goiano \\ Rodovia Geraldo Silva Nascimento, Km 2,5, Zona Rural, CEP 75790-000, Urutaí - GO, Brasil \\ ${ }^{3}$ Programa de Pós-Graduação em Ecologia de Ambientes Aquáticos Continentais \\ Universidade Estadual de Maringá \\ Avenida Colombo, 5790, CEP 87020-900, Maringá - PR, Brasil \\ * Autor para correspondência \\ diogoamaral.ueg@gmail.com
}

Submetido em 30/08/2017

Aceito para publicação em 16/03/2018

\section{Resumo}

Psitacídeos que vivem em cidades podem utilizar as praças urbanas como áreas para reprodução, refúgio ou alimentação. No entanto, existe pouco conhecimento sobre a dieta dessas aves nestes ambientes. Assim, este trabalho teve como objetivos identificar os recursos alimentares explorados por psitacídeos em praças urbanas e verificar as estratégias de forrageamento utilizadas. Foram visitadas 10 praças urbanas da cidade de Uberlândia, MG, Brasil, no período de maio a dezembro de 2011. As aves foram investigadas pelos métodos animal-focal e $a d$ libitum. As observações foram realizadas pela manhã e no final da tarde, totalizando $480 \mathrm{~h}$ de esforço amostral. Foram registrados 278 eventos de alimentação de quatro espécies de psitacídeos: Eupsittula aurea, Psittacara leucophthalmus, Brotogeris chiriri e Diopsittaca nobilis. As aves observadas consumiram recursos alimentares de 33 espécies vegetais, incluindo frutos (somente polpa, polpa junto com semente ou somente semente), flores, néctar e folhas. As famílias de plantas mais exploradas foram Fabaceae, Bignoniaceae e Arecaceae. A dieta dos psitacídeos foi bastante diversificada, com a exploração de uma ampla gama de espécies vegetais, principalmente as exóticas. Isso evidencia a importância das praças urbanas na alimentação, permanência e conservação de algumas espécies de psitacídeos nos centros urbanos.

Palavras-chave: Dieta; Ecologia de aves; Ecologia urbana; Espécies exóticas; Frugivoria

\section{Abstract}

Exploration of food resources by psittacids (Aves: Psittaciformes) in the urban area of Uberlândia, Minas Gerais, Brazil. Parrots living in cities can use urban parks for breeding, refuge or feeding. However, 
there is little knowledge about the diet of parrots in these environments. We identified food resources exploited by parrots in urban public parks and examined the foraging strategies employed. The study was conducted in 10 parks in the city of Uberlândia, MG, Brazil, from May to December 2011. The psittacids were investigated by focal animal and ad libitum methods. Observations were carried out in the morning and late afternoon, totaling 480 hours of sampling effort. A total of 278 feeding events were recorded. The parrot species for which foraging records were obtained were Eupsittula aurea, Psittacara leucophthalmus, Brotogeris chiriri and Diopsittaca nobilis. The parrots consumed food resources from 33 plant species, including fruit (pulp only, pulp with seed or seed only), flowers, nectar and leaves. The most exploited plant families were Fabaceae, Bignoniaceae and Arecaceae. The diet of the psittacids was quite diverse, where they exploited a wide range of plants, especially exotic species. These results demonstrated the importance of green areas for the feeding, refuge and conservation of some parrot species in urban centers.

Key words: Avian ecology; Diet; Exotic species; Frugivory; Urban ecology

\section{Introdução}

A urbanização é um processo capaz de produzir mudanças significativas nas condições químicas, físicas e ecológicas em áreas de desenvolvimento urbano, assim, resultando na criação de uma nova paisagem e novas assembleias de plantas e animais (KINZIG; GROVE, 2001). A urbanização é uma das principais causas da perda de biodiversidade e mudanças ambientais em escala local e global (GRIMM et al., 2008; McPHEARSON et al., 2016). Enquanto algumas espécies podem se habituar facilmente a viver em áreas urbanas, outras, mais sensíveis, são negativamente afetadas pelo processo de urbanização (LUNIAK, 2004). As mudanças bióticas (por ex. presença de espécies exóticas) e abióticas (por ex. aumento da temperatura), assim como as alterações da paisagem, fazem com que espécies habitat especialistas apresentem declínios populacionais ou até mesmo extinções locais, não conseguindo se estabelecer em ambientes afetados pela urbanização (SHOCHAT et al., 2006). No entanto, ambientes urbanos podem conter arvores frutíferas que servem de alimento para aves, que também podem se beneficiar de alimentos de origem antrópica (BARROS et al., 2014). Além disso, espécies de aves migratórias também podem utilizar esses ambientes para repouso (IDILFITRI; MOHAMAD, 2012; CARDILINI et al., 2013).

A família Psittacidae inclui araras, papagaios e periquitos, e possui representantes nas zonas tropicais e subtropicais do planeta (SICK, 1997; FORSHAW, 2010). Na região neotropical, $31 \%$ das espécies de psitacídeos estão sob ameaça de extinção (COLLAR et al., 1994). Fatores como perda de hábitat, caça e tráfico, assim como o aumento da competição e predação como resultado da introdução de espécies exóticas, fazem com que os psitacídeos estejam no topo das listas globais de espécies ameaçadas (COLLAR; JUNIPER, 1992; COLLAR, 2000). Essas aves geralmente possuem baixas taxas de reprodução, baixa sobrevivência de filhotes, longo tempo para atingir a maturidade sexual, e elevadas exigências na escolha de locais para nidificação (COLLAR; JUNIPER, 1992; SNYDER et al., 2000; WRIGHT et al., 2001). No entanto, psitacídeos apresentam diferentes graus de sensibilidade aos impactos antrópicos devido as variações na abundância local das espécies (comuns ou raras), amplitude de distribuição geográfica (ampla ou restrita), exigências de habitat (generalistas ou especialistas), tamanho corporal e dieta (GALETTI et al., 2002).

A dieta da maioria dos psitacídeos é composta por sementes, castanhas e bagas, mas pode incluir outros itens alimentares como néctar, pólen, liquens, fungos, insetos e até mesmo depósitos de argila ricos em minerais (RAGUSA-NETTO; FECCHIO, 2006; FORSHAW, 2010). Os recursos vegetais explorados pelos psitacídeos podem apresentar grande variação temporal e espacial, em termos de abundância, de modo que os bandos se deslocam por grandes distâncias, acompanhando a sua disponibilidade (RENTON, 2001). Embora existam alguns estudos abordando a alimentação de psitacídeos (por ex. KRISTOSCH; MARCONDES-MACHADO, 2001; SILVA, 2005; RAGUSA-NETTO; FECCHIO, 2006), pouco se sabe 
sobre a dieta das espécies neotropicais em ambientes urbanos (por ex. SANTOS; RAGUSA-NETTO, 2014). Psitacídeos são ecologicamente importantes não só por causa de seu papel como predadores de frutos, sementes e flores (GALETTI; RODRIGUES, 1992; SILVA, 2005), mas também como bons dispersores de sementes (BLANCO et al., 2016; BAÑOS-VILLALBA et al., 2017), e consequentemente devido ao seu impacto na estrutura do habitat (SICK, 1997). Além disso, esse grupo constitui uma grande fração da biomassa de aves em muitos ambientes tropicais (TERBORGH et al., 1990). Estudar a dieta, comportamento e ecologia dos psitacídeos é importante pois pode auxiliar nos esforços de reintrodução de espécies ameaçadas de extinção, em projetos de reflorestamento, na compreensão dos processos de controle de dispersão e predação de plantas e principalmente para elaboração de projetos para a conservação de muitas espécies (COLLAR; JUNIPER, 1992; COLLAR, 2000; BLANCO et al., 2016; BAÑOSVILLALBA et al., 2017).
As aves estão envolvidas em diversas funções ecossistêmicas, entre as quais podemos citar a polinização e dispersão de sementes (SILVA; PEDRONI, 2014). Através da dieta das aves pode-se obter informações sobre a estrutura trófica das comunidades, bem como das condições físicas do ambiente (SICK, 1997). Assim, estudar espécies que utilizam áreas antropizadas (áreas verdes urbanas) é uma boa oportunidade para conhecer os efeitos da urbanização sobre as espécies e comunidades, colaborando com a preservação da biodiversidade. Portanto, o presente estudo teve como objetivos: 1) identificar os tipos de recursos alimentares explorados por psitacídeos em praças da área urbana da cidade de Uberlândia, Minas Gerais, Brasil; 2) avaliar a forma e a frequência com que esses itens alimentares são consumidos, e; 3) descrever as estratégias de forrageamento empregadas pelas espécies investigadas.

FIGURA 1: Distribuição das praças amostradas dentro do perímetro urbano de Uberlândia, MG, Brasil. (P1. Praça Urias Batista dos Santos, P2. Praça Ana Diniz, P3. Praça Lincoln, P4. Praça Edgar de Paulo, P5. Praça Feres, P6. Praça Pereira de Rezende, P7. Praça Américo Ferreira de Abreu, P8. Praça Clarimundo Carneiro, P9. Praça Anahyta Tannus Fonseca, P10. Praça Theodora dos Santos).

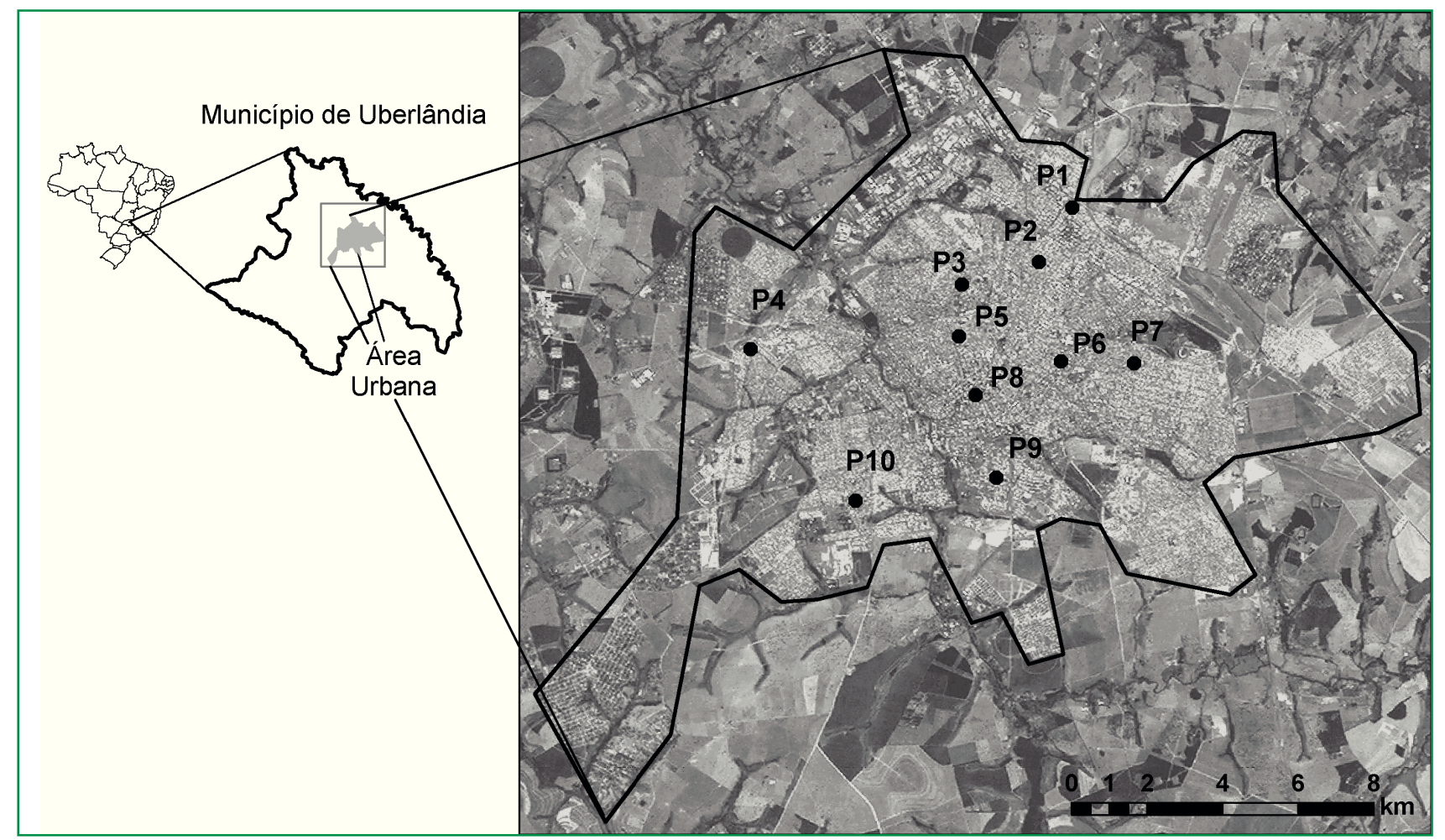




\section{Material e Métodos}

\section{Área de estudo}

$\mathrm{O}$ estudo foi realizado no perímetro urbano do município de Uberlândia (18 $54^{\circ} \mathrm{S}, 48^{\circ} 15^{\prime} \mathrm{W}$; Figura 1), localizado na Mesorregião do Triângulo Mineiro/Alto Paranaíba, no estado de Minas Gerais, região Sudeste do Brasil (SECRETARIA MUNICIPAL DE PLANEJAMENTO URBANO, 2011). A área total do município é de $4.115,206 \mathrm{~km}^{2}$, com uma população de 604.013 pessoas, 97\% das quais residentes na área urbana (IBGE, 2016).

A cidade está situada no domínio dos Planaltos e Chapadas da Bacia Sedimentar do Paraná, inserida no bioma Cerrado (SECRETARIA MUNICIPAL DE PLANEJAMENTO URBANO, 2011). De acordo com a classificação de Köppen, o clima é tropical de altitude (Cwa), marcado por uma estação chuvosa e quente (outubro a abril) e outra seca e amena (maio a setembro) (PEEL et al., 2007). As médias mensais de temperatura durante os meses de execução da pesquisa variaram de $19,9^{\circ} \mathrm{C}$ a $24,7^{\circ} \mathrm{C}$, enquanto a precipitação mensal máxima durante a estação seca foi de 4,8 $\mathrm{mm}$ e de 253,7 mm na estação chuvosa (PREFEITURA MUNICIPAL DE UBERLÂNDIA, 2011).

Foram selecionadas 10 praças urbanas da cidade de Uberlândia, a partir de dados de ocorrência de espécies de psitacídeos (MARQUES, 2010). Os critérios de escolha foram: riqueza e abundância de psitacídeos, área da praça urbana e presença de diferentes espécies vegetais (Figura 1; Tabela 1).

\section{Procedimentos}

Todas as praças foram visitadas uma vez por mês entre Maio e Dezembro de 2011. Cada visita foi realizada por dois pesquisadores, mas, para manter a padronização dos registros, um dos pesquisadores esteve presente em todas as visitas. As observações ocorreram no horário de maior atividade dos psitacídeos, das 07h00min até as $10 \mathrm{~h} 00 \mathrm{~min}$ e das $16 \mathrm{~h} 00 \mathrm{~min}$ às $19 \mathrm{~h} 00 \mathrm{~min}$, com um total de $480 \mathrm{~h}$ de amostragem (48 h em cada praça). Para os registros visuais foram usados binóculos Nikon (8x40), através dos métodos animal-focal e ad libitum (ALTMAN, 1974). Os registros foram realizados quando um indivíduo era observado se alimentando. Considerouse como evento de alimentação (EA) o registro feito de cada indivíduo ou por um grupo de indivíduos da mesma espécie de psitacídeo consumindo determinado item alimentar de uma dada espécie vegetal. Durante as observações foram registrados: (i) a espécie de psitacídeo, (ii) o número de indivíduos de cada espécie, (iii) espécie vegetal utilizada, (iv) horário de chegada e de saída dos indivíduos, (v) o item alimentar consumido (polpa, folha, semente, flor, néctar e botão da flor), e a (vi) forma como o alimento foi manipulado.

Os padrões comportamentais para a manipulação dos itens alimentares foram classificados com base nos estudos prévios de Moermond e Denslow (1985) e Kristosch e Marcondes-Machado (2001): sendo:

TABELA 1: Coordenadas geográficas e área $\left(\mathrm{m}^{2}\right)$ das praças urbanas amostrados na cidade de Uberlândia, MG.

\begin{tabular}{|c|c|c|c|}
\hline Praças & Latitude & Longitude & Área $\left(\mathrm{m}^{2}\right)$ \\
\hline Praça Urias Batista Santos & $18^{\circ} 52^{\prime} 43.54 ” \mathrm{~S}$ & $48^{\circ} 15^{\prime} 31.53^{\prime \prime} \mathrm{O}$ & 8.960 \\
\hline Praça Ana Diniz & $18^{\circ} 53^{\prime} 30.20^{\prime \prime} \mathrm{S}$ & $48^{\circ} 16^{\prime} 0.03{ }^{\prime \prime O}$ & 8.160 \\
\hline Praça Américo Ferreira de Abreu & $18^{\circ} 54^{\prime} 57.20^{\prime \prime} \mathrm{S}$ & $48^{\circ} 14^{\prime} 38.65^{\prime \prime} \mathrm{O}$ & 15.230 \\
\hline Praça Rubens Pereira de Rezende & $18^{\circ} 54^{\prime} 55.46^{\prime \prime} \mathrm{S}$ & $48^{\circ} 15^{\prime} 41.03^{\prime \prime O}$ & 5.700 \\
\hline Praça Loncoln & $18^{\circ} 53^{\prime} 49.74 ” \mathrm{~S}$ & $48^{\circ} 17^{\prime} 6.03{ }^{\prime \prime O}$ & 7.035 \\
\hline Praça Nicolau Feres & $18^{\circ} 54^{\prime} 34.10^{\prime \prime} \mathrm{S}$ & $48^{\circ} 17^{\prime} 8.55^{\prime \prime} \mathrm{O}$ & 11.370 \\
\hline Praça Clarimundo Carneiro & $18^{\circ} 55^{\prime} 24.64^{\prime \prime} \mathrm{S}$ & $48^{\circ} 16^{\prime} 53.89^{\prime \prime} \mathrm{O}$ & 7.880 \\
\hline Praça Anahyta Tannus Fonseca & $18^{\circ} 56^{\prime} 35.93^{\prime \prime S}$ & $48^{\circ} 16^{\prime} 36.63^{\prime \prime O}$ & 8.827 \\
\hline Praça Theodora dos Santos & $18^{\circ} 56^{\prime} 55.43^{\prime \prime S}$ & $48^{\circ} 18^{\prime} 36.83^{\prime \prime O}$ & 10.380 \\
\hline Praça Edgar de Paulo & $18^{\circ} 54^{\prime} 45.24 ” S$ & $48^{\circ} 20^{\prime} 6.80^{\prime \prime} \mathrm{O}$ & 7.570 \\
\hline
\end{tabular}


i) "bicadas no alimento", quando a ave remove com o bico pedaços do item alimentar sem arrancá-lo da planta, sendo tais pedaços engolidos logo em seguida, ou previamente triturados para posteriormente engolir; ii) "arranca e tritura o alimento", quando a ave pega o item alimentar com o bico, removendo-o inteiro da planta, e o tritura antes de engolir; iii) "arranca o alimento e segura com o pé" triturando o alimento em seguida; iv) “consumo do néctar", quando a ave consome o néctar do fruto sem triturá-lo por inteiro - neste método, nem sempre o fruto é arrancado -, e por último o método v) "pé como pinça", a ave utiliza o pé como auxílio durante todo o consumo do alimento.

Como os Psitacídeos apresentam o comportamento de lateralidade ao manipular os itens alimentares (SICK, 1997; MAGAT; BROWN, 2009; DUGGAN et al., 2016), foi observado se as espécies possuem preferência pela utilização de um dos pés (direito ou esquerdo) durante a alimentação. O termo "auxílio com o pé" foi empregado quando o indivíduo usava o pé para facilitar a manipulação do alimento, ou para retirar itens alimentares do bico, de forma que pudessem terminar de consumi-los.

A nomenclatura e a ordem taxonômica das espécies de psitacídeos estão de acordo com o Comitê Brasileiro de Registros Ornitológicos (PIACENTINI et al., 2015). As espécies vegetais consumidas foram coletadas, identificadas segundo Lorenzi (2002) e Lorenzi et al. (2003) e depositadas no Herbarium Uberlandensis (HUFU) da Universidade Federal de Uberlândia, como material-testemunho. A nomenclatura e a ordem taxonômica das espécies vegetais seguiram "Tropicos: botanical information system" (MISSOURI BOTANICAL GARDEN, 2010).

\section{Resultados}

\section{Dieta e comportamento alimentar das espécies de psitacídeos}

Foram registrados 278 eventos de alimentação (EA) das seguintes espécies de psitacídeos: Eupsittula aurea (Gmelin, 1788), Psittacara leucophthalmus
(Statius Muller, 1776), Brotogeris chiriri (Vieillot, 1818), Diopsittaca nobilis (Linnaeus, 1758) e Forpus xanthopterygius (Spix, 1824). Durante cada amostragem (sessão de observação) foram observados uma média de 10,92 $\pm 12,17$ (média \pm desvio padrão) indivíduos de Brotogeris chiriri, 3,32 44,63 indivíduos de $P$. leucophthalmus, 0,33 $\pm 0,92$ indivíduos de D. nobilis, $0,24 \pm 0,62$ indivíduos de $E$. aurea e $0,11 \pm 0,54$ indivíduos de $F$. xanthopterygius. Somente $F$. xanthopterygius não foi registrado se alimentando. Brotogeris chiriri foi a espécie com maior número de EA $(217 ; 78,06 \%)$, seguida por P. leucophthalmus $(37 ; 13,31 \%)$, D. nobilis $(17 ; 6,11 \%)$ e E. aurea (sete; $2,52 \%$ ) (Tabela 2).

Trinta e quatro espécies vegetais foram exploradas pelos Psitacídeos, as quais se encontram distribuídas em 12 famílias. Algumas espécies foram consumidas por mais de uma espécie de psitacídeo: Morus nigra (Moraceae) e Terminalia catappa (Combretaceae) (consumidas por quatro espécies); Syzygium cumini (Myrtaceae) e Triplaris americana (Poligonaceae) (consumidas por três espécies); e Cassia fistula (Fabaceae), Ficus sp. (Moraceae), Handroanthus sp. (Bignoniaceae), Jacaranda mimosifolia (Bignoniaceae), Mangifera indica (Anacardiaceae), Livistona chinensis (Arecaceae), Phoenix canariensis (Arecaceae), Tectona grandis (Lamiaceae) e Astronium urundeuva (Anacardiaceae) (consumidas por duas espécies) (Tabela 2).

Entre as espécies de psitacídeos registradas, $B$. chiriri foi a que explorou o maior número de espécies vegetais na sua dieta (29 espécies; 85,29\%), seguido por P. leucophthalmus (10 espécies; 29,41\%), D. nobilis (nove espécies; 26,47\%) e E. aurea (cinco espécies; 14,70\%). Brotogeris chiriri e P. leucophthalmus foram as espécies que consumiram o maior número de itens alimentares (seis itens cada), seguidos por D. nobilis (cinco itens) e E. aurea (quatro itens) (Tabela 2).

O consumo de néctar foi observado para $P$. leucophthalmus e para B. chiriri. Geralmente, os indivíduos dessas espécies pegavam a flor com o bico (sem tocar a parte reprodutiva), destacavam do pedicelo, perfuravam o cálice para sorver o néctar e em seguida descartavam a flor sob a árvore. Brotogeris chiriri consumiu o néctar de Spathodea campanulata e Delonix 
regia, inserindo o bico nas flores (sem arrancá-las pelos pedicelos), e de Handroanthus spp., D. regia e Erythrina speciosa, removendo as flores com o bico (segurandoas com um dos pés e macerando a base da flor onde se encontrava o nectário). Este último modo de consumo foi classificado como "arranca e segura com o pé" ou "auxílio com o pé". Durante o consumo do néctar de Handroanthus spp., os indivíduos de $P$. leucophthalmus manipulavam as flores de forma mais lenta que os indivíduos de $B$. chiriri e às vezes utilizavam um dos pés como auxílio para segurar a flor.

\section{Dieta e comportamento alimentar de Eupsittula aurea}

O item alimentar mais consumido por E. aurea foi semente $(\mathrm{N}=3 ; 42,85 \%)$, seguido por polpa $(\mathrm{N}=$ $2 ; 28,57 \%$ ) e por polpa/semente e folha, que foram consumidos na mesma proporção $(\mathrm{N}=1 ; 14,28 \%$ cada $)$ (Tabela 2). O consumo de espécies vegetais da família Fabaceae representou $28,57 \%$ do total de EA de $E$. aurea. Das espécies preferenciais para alimentação deste psitacídeo três delas são exóticas (Leucaena leucocephala, Morus nigra e Terminalia catappa), representando $71 \%$ da alimentação observada (Tabela 2 ).

TABELA 2: Espécies vegetais consumidas pelas diferentes espécies de psitacídeos no período de maio a dezembro de 2011 nas praças urbanas da cidade de Uberlândia, MG, Brasil. Item: Pol - polpa; Fol - folha; Sem - semente; Flo - flor; Nec - néctar; Bot - botão de flor; Método: B - bicadas no alimento; T - arranca e tritura o alimento; $\mathrm{S}$ - arranca o alimento, segura-o com o pé e come; $\mathrm{P}$ - consumo de néctar; A - o pé é utilizado como auxílio durante o consumo do alimento; Registros: número de eventos de alimentação; \%EA: porcentagem de eventos de alimentação, calculada em relação ao número total de eventos de alimentação de cada espécie de psitacídeo. Os asteriscos indicam espécies vegetais exóticas.

\begin{tabular}{|c|c|c|c|c|c|c|}
\hline Psitacídeo & $\begin{array}{c}\text { Família da } \\
\text { planta }\end{array}$ & Espécie da planta & Item & Método & Registros & \%EA \\
\hline \multicolumn{7}{|l|}{ Eupsittula aurea } \\
\hline & \multirow{2}{*}{ Fabaceae } & Plathymenia reticulata Benth. & Sem & $\mathrm{S}$ & 1 & $14,28 \%$ \\
\hline & & Leucaena leucocephala (Lam.) de Wit* & Sem & $\mathrm{S} ; \mathrm{T}$ & 1 & $14,28 \%$ \\
\hline & Moraceae & Morus nigra $\mathrm{L} . *$ & $\mathrm{Pol} / \mathrm{Sem}$ & $\mathrm{T}$ & 1 & $14,28 \%$ \\
\hline & Anacardiaceae & Astronium urundeuva (Allemão) Engl. & Sem & $\mathrm{T}$ & 1 & $14,28 \%$ \\
\hline & \multirow{2}{*}{ Combretaceae } & Terminalia catappa $\mathrm{L} *^{*}$ & Fol & $\mathrm{S}$ & 1 & $14,28 \%$ \\
\hline & & Terminalia catappa $\mathrm{L} . *$ & Pol & S; B & 2 & $28,57 \%$ \\
\hline \multicolumn{7}{|l|}{$\begin{array}{l}\text { Psittacara } \\
\text { leucophthalmus }\end{array}$} \\
\hline & Mytaceae & Syzygium cumini (L.) Skeels* & Pol & $\mathrm{S} ; \mathrm{T} ; \mathrm{B} ; \mathrm{A}$ & 5 & $13,51 \%$ \\
\hline & & Cassia fistula $\mathrm{L} . *$ & Flo & $\mathrm{T}$ & 1 & $2,70 \%$ \\
\hline & Moraceae & Ficus sp. & $\mathrm{Pol} / \mathrm{Sem}$ & $\mathrm{T}$ & 3 & $8,11 \%$ \\
\hline & & Morus nigra L.* & $\mathrm{Pol} / \mathrm{Sem}$ & $\mathrm{T}$ & 2 & $5,41 \%$ \\
\hline & Bignoniaceae & Handroanthus spp. & $\mathrm{Nec}$ & $\mathrm{P} ; \mathrm{A}$ & 4 & $10,81 \%$ \\
\hline & & Jacaranda mimosifolia D. Don* & $\mathrm{Nec}$ & $\mathrm{P}$ & 1 & $2,70 \%$ \\
\hline & Anacardiaceae & Mangifera indica $\mathrm{L} . *$ & Pol & B & 1 & $2,70 \%$ \\
\hline & & Astronium urundeuva (Allemão) Engl. & Sem & $\mathrm{T}$ & 5 & $13,51 \%$ \\
\hline & & Terminalia catappa $\mathrm{L} . *$ & Pol & $\mathrm{S} ; \mathrm{B}$ & 8 & $21,62 \%$ \\
\hline & Combretaceae & Terminalia catappa $\mathrm{L} . *$ & Flo & $\mathrm{T} ; \mathrm{B}$ & 3 & $8,11 \%$ \\
\hline & & Terminalia catappa L.* & Fol & S; B & 2 & $5,41 \%$ \\
\hline & Polygonaceae & Triplaris americana $\mathrm{L}$. & Flo & $\mathrm{T}$ & 2 & $5,41 \%$ \\
\hline
\end{tabular}




\begin{tabular}{|c|c|c|c|c|c|c|}
\hline Psitacídeo & $\begin{array}{c}\text { Família da } \\
\text { planta }\end{array}$ & Espécie da planta & Item & Método & Registros & $\% \mathbf{E A}$ \\
\hline \multicolumn{7}{|c|}{ Brotogeris chiriri } \\
\hline & \multirow{7}{*}{ Arecaceae } & Arecaceae 1 & Flo & $\mathrm{T}$ & 11 & $5,07 \%$ \\
\hline & & Arecaceae 1 & Pol & B & 1 & $0,46 \%$ \\
\hline & & Arecaceae 2 & Pol & $\mathrm{S} ; \mathrm{T} ; \mathrm{B}$ & 2 & $0,92 \%$ \\
\hline & & $\begin{array}{l}\text { Livistona chinensis (Jacq.) R. Bc. ex } \\
\text { Mart.* }\end{array}$ & Flo & $\mathrm{T}$ & 3 & $1,38 \%$ \\
\hline & & $\begin{array}{l}\text { Livistona chinensis (Jacq.) R. Bc. ex } \\
\text { Mart.* }\end{array}$ & Pol & $\mathrm{S} ; \mathrm{B}$ & 15 & $6,91 \%$ \\
\hline & & Phoenix canariensis Chabaud* & Pol & $\mathrm{S} ; \mathrm{B}$ & 4 & $1,48 \%$ \\
\hline & & Syagrus sp. & Flo & $\mathrm{S} ; \mathrm{T}$ & 18 & $8,29 \%$ \\
\hline & \multirow{3}{*}{ Mytaceae } & Syagrus sp. & Pol & $\mathrm{S} ; \mathrm{B}$ & 8 & $3,69 \%$ \\
\hline & & $\begin{array}{l}\text { Callistemon viminalis (Sol. ex Gaertn.) } \\
\text { G. Don ex Loudon* }\end{array}$ & $\mathrm{Nec}$ & $P$ & 1 & $0,46 \%$ \\
\hline & & Syzygium cumini (L.) Skeels* & Pol & S & 42 & $19,35 \%$ \\
\hline & \multirow{9}{*}{ Fabaceae } & $\begin{array}{l}\text { Caesalpinia pluviosa var. peltophoroides } \\
\text { (Benth.) G.P. Lewis }\end{array}$ & Flo & $\mathrm{S} ; \mathrm{T} ; \mathrm{B} ; \mathrm{A}$ & 3 & $1,38 \%$ \\
\hline & & Clitoria fairchildiana R.A. Howard & $\mathrm{Nec}$ & $\mathrm{P} ; \mathrm{A}$ & 1 & $0,46 \%$ \\
\hline & & Delonix regia (Bojer ex Hook.) Raf.* & $\mathrm{Nec}$ & $\mathrm{S} ; \mathrm{P} ; \mathrm{A}$ & 3 & $1,38 \%$ \\
\hline & & Erythrina speciosa Andrews & $\mathrm{Nec}$ & $\mathrm{S} ; \mathrm{P}$ & 3 & $1,38 \%$ \\
\hline & & Inga edulis var. edulis Mart. & $\mathrm{Nec}$ & $\mathrm{P}$ & 6 & $2,76 \%$ \\
\hline & & Peltophorum dubium (Spreng.) Taub. & Flo & $\mathrm{S} ; \mathrm{T} ; \mathrm{A}$ & 1 & $0,46 \%$ \\
\hline & & Peltophorum dubium (Spreng.) Taub. & Flo/Bot & $\mathrm{S} ; \mathrm{T} ; \mathrm{A}$ & 3 & $1,38 \%$ \\
\hline & & Senna sp. & Fol & $\mathrm{S}$ & 1 & $0,46 \%$ \\
\hline & & Senna sp. & Flo/Bot & $\mathrm{S} ; \mathrm{A}$ & 1 & $0,46 \%$ \\
\hline & & Cassia fistula L.* & Flo & $\mathrm{S}$ & 1 & $0,46 \%$ \\
\hline & \multirow{4}{*}{ Malvaceae } & Chorisia speciosa A. St.-Hil. & $\mathrm{Nec}$ & $\mathrm{P}$ & 1 & $0,46 \%$ \\
\hline & & Chorisia speciosa A. St.-Hil. & Sem & $\mathrm{T}$ & 5 & $2,30 \%$ \\
\hline & & Chorisia speciosa A. St.-Hil. & Fol & $\mathrm{S}$ & 1 & $0,46 \%$ \\
\hline & & Pachira aquatica Aubl. & Flo/Bot & $\mathrm{B}$ & 1 & $0,46 \%$ \\
\hline & \multirow{3}{*}{ Moraceae } & Pachira aquatica Aubl. & Sem & $\mathrm{S} ; \mathrm{B}$ & 4 & $1,84 \%$ \\
\hline & & Ficus sp. & $\mathrm{Pol} / \mathrm{Sem}$ & $\mathrm{T}$ & 1 & $0,46 \%$ \\
\hline & & Morus nigra $\mathrm{L} . *$ & $\mathrm{Pol} / \mathrm{Sem}$ & $\mathrm{T}$ & 1 & $0,46 \%$ \\
\hline & \multirow{7}{*}{ Bignoniaceae } & Handroanthus spp. & $\mathrm{Nec}$ & $\mathrm{S} ; \mathrm{P}$ & 25 & $11,52 \%$ \\
\hline & & Handroanthus spp. & Sem & $\mathrm{S} ; \mathrm{T}$ & 3 & $1,38 \%$ \\
\hline & & Jacaranda mimosifolia D. Don* & $\mathrm{Nec}$ & $\mathrm{P}$ & 2 & $0,92 \%$ \\
\hline & & Jacaranda mimosifolia D. Don* & Fol & $\mathrm{B}$ & 1 & $0,46 \%$ \\
\hline & & Spathodea campanulata P. Beauv.* & $\mathrm{Nec}$ & $\mathrm{P}$ & 1 & $0,46 \%$ \\
\hline & & Spathodea campanulata P. Beauv.* & Sem & $\mathrm{S} ; \mathrm{T}$ & 4 & $1,84 \%$ \\
\hline & & Pyrostegia venusta (Ker Gawl.) Miers & $\mathrm{Nec}$ & $\mathrm{P}$ & 1 & $0,46 \%$ \\
\hline & \multirow{3}{*}{ Chrysobalanaceae } & Licania tomentosa (Benth.) Fritsch & Pol & $\mathrm{B}$ & 1 & $0,46 \%$ \\
\hline & & Licania tomentosa (Benth.) Fritsch & Fol & $\mathrm{S} ; \mathrm{T}$ & 1 & $0,46 \%$ \\
\hline & & Licania tomentosa (Benth.) Fritsch & Fol/Bot & $\mathrm{S} ; \mathrm{T}$ & 1 & $0,46 \%$ \\
\hline & Anacardiaceae & Mangifera indica $\mathrm{L} . *$ & Pol & $\mathrm{B}$ & 8 & $3,69 \%$ \\
\hline
\end{tabular}




\begin{tabular}{|c|c|c|c|c|c|c|}
\hline Psitacídeo & $\begin{array}{c}\text { Família da } \\
\text { planta }\end{array}$ & Espécie da planta & Item & Método & Registros & $\% \mathbf{E A}$ \\
\hline & Muntigiaceae & Muntingia calabura L. * & $\mathrm{Pol} / \mathrm{Sem}$ & $\mathrm{S} ; \mathrm{T}$ & 3 & $1,38 \%$ \\
\hline & Lamiaceae & Tectona grandis L. f.* & Sem & $\mathrm{S}$ & 1 & $0,46 \%$ \\
\hline & \multirow{3}{*}{ Combretaceae } & Terminalia catappa $\mathrm{L} . *$ & Pol & $\mathrm{S} ; \mathrm{B}$ & 10 & $4,61 \%$ \\
\hline & & Terminalia catappa $\mathrm{L} . *$ & Flo & $\mathrm{S} ; \mathrm{T}$ & 6 & $2,76 \%$ \\
\hline & & Terminalia catappa L.* & Fol & $\mathrm{B}$ & 4 & $1,84 \%$ \\
\hline & \multirow{2}{*}{ Polygonaceae } & Triplaris americana $\mathrm{L}$. & Flo & $\mathrm{T}$ & 2 & $0,92 \%$ \\
\hline & & Triplaris americana $\mathrm{L}$. & Bot & $\mathrm{S} ; \mathrm{B}$ & 1 & $0,46 \%$ \\
\hline \multicolumn{7}{|l|}{$\begin{array}{l}\text { Diopsittaca } \\
\text { nobilis }\end{array}$} \\
\hline & \multirow{3}{*}{ Arecaceae } & $\begin{array}{l}\text { Livistona chinensis (Jacq.) R. Bc. ex } \\
\text { Mart.* }\end{array}$ & Pol & $\mathrm{S} ; \mathrm{T}$ & 5 & $29,41 \%$ \\
\hline & & Phoenix canariensis Chabaud* & Pol & $\mathrm{T}$ & 1 & $5,88 \%$ \\
\hline & & Syzygium cumini (L.) Skeels* & Flo & $\mathrm{T}$ & 1 & $5,88 \%$ \\
\hline & Mytaceae & Psidium guajava $\mathrm{L}$. & $\mathrm{Pol} / \mathrm{Sem}$ & B & 1 & $5,88 \%$ \\
\hline & Fabaceae & Tipuana tipu (Benth.) Kuntze* & Sem & $\mathrm{S}$ & 1 & $5,88 \%$ \\
\hline & Moraceae & Morus nigra L.* & $\mathrm{Pol} / \mathrm{Sem}$ & $\mathrm{T}$ & 1 & $5,88 \%$ \\
\hline & Lamiaceae & Tectona grandis L. f.* & Sem & $\mathrm{S}$ & 2 & $11,76 \%$ \\
\hline & \multirow{2}{*}{ Combretaceae } & Terminalia catappa $\mathrm{L} . *$ & Pol & $\mathrm{S} ; \mathrm{T} ; \mathrm{B}$ & 3 & $17,65 \%$ \\
\hline & & Terminalia catappa $\mathrm{L} . *$ & Fol & B & 1 & $5,88 \%$ \\
\hline & Polygonaceae & Triplaris americana L. & Flo & $\mathrm{T}$ & 1 & $5,88 \%$ \\
\hline
\end{tabular}

Terminalia catappa (Combretaceae) destacou-se por ter sido a espécie vegetal com o maior número de itens alimentares e EA observados (Tabela 2). Quanto aos métodos de manipulação do alimento, E. aurea utilizou o "arranca e segura com o pé" com maior frequência ( $50 \%$ do total de registros para esta espécie), com destaque para a utilização do pé direito (60\%), seguido pelo método "arranca e tritura" $(30 \%)$ e, por fim, o método "bicadas" $(20 \%)$.

\section{Dieta e comportamento alimentar de Psittacara leucophthalmus}

O item alimentar mais consumido por $P$. leucophthalmus foi polpa ( $\mathrm{N}=14 ; 37,83 \%)$, seguido pelo consumo de flor $(\mathrm{N}=6 ; 16,22 \%)$, polpa, polpa/ semente e semente $(\mathrm{N}=5 ; 13,52 \%$ cada), e por último o consumo de folha $(\mathrm{N}=2 ; 5,40 \%)$ (Tabela 2$)$. As famílias vegetais com o maior número de espécies representadas na dieta foram Anacardiaceae, Bignoniaceae e Moraceae, com duas espécies $(20 \%$ do total de itens, cada), as demais famílias apresentaram apenas uma espécie. Entre as espécies vegetais preferenciais para a alimentação desta espécie nas praças urbanas, seis são exóticas, representando $66,67 \%$ das plantas consumidas (Tabela 2).

Três itens alimentares (polpa, flor e folha jovem) foram consumidos de Terminalia catapa, enquanto para outras espécies vegetais, apenas um tipo de item alimentar foi consumido. Além disso, T. catappa foi a espécie que apresentou o maior número de EA (N $=13 ; 35,14 \%)$, seguida por Syzygium cumini $(\mathrm{N}=5$; $13,51 \%)$ e Handroanthus spp. $(\mathrm{N}=4 ; 10,81 \%)$ (Tabela 2). Em relação aos métodos de manipulação dos itens alimentares por $P$. leucophthalmus, "arranca e tritura" foi o mais utilizado ( $46,03 \%$ do total de registros), seguido por "bicadas" $(20,64 \%)$, "arranca e segura com o pé" $(20,63 \%)$, e, por fim, "auxílio com o pé" $(12,69 \%)$. Os indivíduos utilizaram ambos os pés para segurar o alimento, com a mesma frequência (50,0\% cada). 


\section{Dieta e comportamento alimentar de Brotogeris chiriri}

O principal item alimentar consumido por $B$. chiriri foi polpa $(\mathrm{N}=91 ; 41,93 \%)$, seguido por flor $(\mathrm{N}=45$; $20,73 \%)$, néctar $(\mathrm{N}=44 ; 20,27 \%)$, semente $(\mathrm{N}=17$; $7,83 \%)$, folha $(\mathrm{N}=8 ; 3,68 \%)$, polpa/semente e flor/ botão $(\mathrm{N}=5 ; 2,30 \%$, cada) e por último folha/botão e botão ( $\mathrm{N}=1 ; 0,47 \%$, cada) (Tabela 2 ). Em relação a importância total de espécies vegetais na dieta de $B$. chiriri, as famílias mais importantes foram Fabaceae $(\mathrm{N}=$ 8; 27,59\%), Bignoniaceae e Arecaceae ( $=4,13,79 \%$ cada). O consumo de espécies da família Arecaceae representou $28,54 \%$ do total de EA e o consumo de espécies da família Bignoniaceae representou 17,04\% do total de EA. A maioria das espécies vegetais consumidas por esta espécie foi de origem exótica $(\mathrm{N}=13 ; 54,17 \%)$ (Tabela 2).

Brotogeris chiriri consumiu os mesmos itens alimentares que $P$. leucophthalmus na espécie vegetal Terminalia catapa demonstrando a importância desta planta para a dieta de ambas as espécies, podendo haver sobreposição na dieta. O comportamento de remover parte de cupinzeiros para posteriormente consumir os cupins também foi observado em duas ocasiões. Quanto aos métodos utilizados por $B$. chiriri na manipulação do alimento, "arranca e segura com o pé" foi o mais utilizado (59,90\% do total de métodos de manipulação), seguido por "bicadas" (21,04\%), "arranca e tritura" $(16,34 \%)$ e "auxílio com o pé” (2,72\%). Brotogeris chiriri utilizou o pé esquerdo com maior frequência $(72,73 \%)$ para segurar os itens alimentares.

\section{Dieta e comportamento alimentar de Diopsittaca nobilis}

O principal item alimentar da dieta de D. nobilis foi polpa $(\mathrm{N}=9 ; 52,94 \%)$, seguido por semente $(\mathrm{N}=3$; $17,65 \%)$, polpa/semente e flor $(\mathrm{N}=2 ; 11,76 \%$ cada $)$ e folha $(\mathrm{N}=1 ; 5,88 \%$ ) (Tabela 2$)$. As famílias com o maior número de espécies vegetais na dieta de $D$. nobilis foram Arecaceae e Myrtaceae ( $\mathrm{N}=2 ; 22,22 \%$ do total de cada família). O consumo de espécies da família Arecaceae representou $35,29 \%$ do total de EA, enquanto o consumo de espécies da família Myrtaceae representou 11,76\% do total de EA. Sete espécies vegetais que compuseram a dieta de $D$. nobilis são exóticas (77,78\%). Livistona chinensis foi a espécie vegetal com o maior número de EA $(29,41 \%)$, seguida por Terminalia catappa $(23,52 \%)$ e Tectona grandis $(11,76 \%)$.

Quanto aos métodos utilizados na manipulação do alimento, D. nobilis utilizou o método "arranca e segura com o pé" com maior frequência ( $60 \%$ do total de métodos de manipulação de alimento), seguido pelo método "arranca e tritura" (30\%) e, por fim, o método "bicadas" (10\%), sendo que não houve diferença na utilização do pé esquerdo ou direito para segurar o item alimentar (50,0\% cada).

\section{Discussão}

Os psitacídeos registrados fazem uso de diferentes estratégias de forrageamento, apresentando uma dieta ampla, na qual exploram diferentes tipos de recursos alimentares nas praças urbanas. As espécies observadas neste estudo já haviam sido observados em outros estudos sobre a avifauna urbana do município de Uberlândia (FRANCHIN; MARÇAL JÚNIOR, 2002; TORGA et al., 2007; VALADÃO et al., 2006a; 2006b), demonstrando que estes psitacídeos vêm se mantendo no ambiente urbano há quase uma década, explorando os recursos disponibilizados pelas áreas verdes da cidade. As praças urbanas são tidas como importantes para a conservação da biodiversidade porque podem servir como áreas para repouso, reprodução e alimentação de algumas espécies da fauna (BENSUSAN, 2006). Neste sentido, aves que apresentam uma dieta mista, como os psitacídeos (RAGUSA-NETTO; FECCHIO, 2006; PARRINI; RAPOSO, 2008; NUNES; SANTOS JUNIOR, 2011), podem ser favorecidos pela presença de árvores frutíferas, que oferecem uma ampla variedade de itens alimentares.

Neste estudo, a polpa foi o item alimentar mais importante para a maioria das espécies de psitacídeos, sendo a exceção dada por E. aurea, que teve a semente como o principal item alimentar. Janzen (1981) argumenta que psitacídeos raramente são frugívoros e geralmente predam as sementes, sendo importantes dispersores de algumas espécies de plantas com sementes pequenas. 
A predação de espécies vegetais é de tal relevância que pode ser observada em diversos estudos que abordam a dieta dos psitacídeos (GALETTI, 1993; KRISTOSCH; MARCONDES-MACHADO, 2001; SILVA, 2005; RAGUSA-NETTO; FECCHIO, 2006). Trabalhos recentes tem demonstrado a importância dos psitacídeos como dispersores endozoocóricos e independentemente da capacidade de germinação aumentada ou limitada após o trânsito intestinal, uma proporção relativamente grande de sementes podem ser encontradas nas fezes dessas aves (BLANCO et al., 2016), demonstrando o seu papel fundamental na formação da estrutura da paisagem (BAÑOS-VILLALBA et al., 2017).

Estudos da dieta de psitacídeos obtiveram resultados variados com relação à quantidade de interações entre as aves e as espécies vegetais (PIZO et al., 1995; GALETTI, 1997; KRISTOSCH; MARCONDESMACHADO, 2001; RAGUSA; FECCHIO, 2006; FONSECA; ANTUNES, 2007). Esta variação nas interações pode estar relacionada com a composição de espécies vegetais da área estudada, quantidade de competidores pelo mesmo recurso alimentar, presença de predadores, disponibilidade de abrigos, distância entre os recursos e locais de repouso e até mesmo com o tempo de estudo despendido para o levantamento da dieta dos psitacídeos (LUCCAS et al., 2009). Além disso, a diferença referente ao uso das espécies vegetais pelos psitacídeos também pode estar relacionada com a composição florística das áreas ou mesmo a preferências de cada espécie (PARANHOS et al., 2007; DA SILVA, 2013). Por exemplo, apesar de papagaios habitualmente se alimentarem de frutos e sementes, o consumo de flores pode ser vital quando diminui a produção de frutas, principalmente durante a estação seca (GALETTI, 1993; RAGUSA-NETTO, 2004; 2005). Embora o registro do consumo de insetos por psitacídeos neotropicais seja relativamente escasso (DEL HOYO et al., 1997; RENTON, 2001), o consumo de cupins já foi observado em E. aurea (PARANHOS et al., 2009) e G. guarouba (SILVA, 1990). As espécies de psitacídeos podem utilizar os cupins como alimento devido a acessibilidade deste item durante a construção e ocupação dos cupinzeiros na época reprodutiva, ou mesmo como suplementação protéica (PARANHOS et al., 2009).
Brotogeris chiriri e P. leucophthalmus realizaram o consumo de néctar sem tocar as partes reprodutivas da planta. Este comportamento é denominado de pilhagem, e já foi observado no consumo de néctar por outros psitacídeos (LARA; ORNELAS, 2001; RAGUSANETTO, 2002; ABREU; VIEIRA, 2004; PARRINI; RAPOSO, 2008). A pilhagem é uma desvantagem para a planta, uma vez que os organismos pilhadores (e.g. aves) consomem o néctar das flores sem necessariamente polinizá-las (CHITTKA; THOMSON, 2004). Paranhos et al. (2007) salientaram que $B$. chiriri não consome todas as estruturas das flores. Neste estudo não foi possível determinar quais estruturas florais foram consumidas, e o néctar pode ter sido o principal recurso procurado, havendo ingestão involuntária de outras partes florais. Embora a utilização de flores para a alimentação de psitacídeos já tenha sido observada (ROTH, 1984), as flores não são um alimento muito procurando pelos psitacídeos, sendo seu consumo mais comum nos períodos de seca, refletindo maior disponibilidade deste recurso alimentar nesta época do ano (PARANHOS et al., 2009). Foi possível relatar o consumo de folhas por E. aurea, P. leucophtaumus, B. chiriri e D. nobilis. $\mathrm{O}$ consumo de folhas por psitacídeos foi relatado pela primeira vez em Pyrrhura frontalis (KRISTOSCH; MARCONDES-MACHADO, 2001), sendo este tipo de alimentação ainda pouco conhecido para este grupo.

A dieta dos Psittacidae registrados neste estudo foi composta essencialmente por espécies vegetais exóticas. Esse resultado provavelmente é consequência do elevado número dessas espécies em áreas verdes urbanas do município de Uberlândia. Cerca de 56,67\% das espécies utilizadas na arborização de um dos bairros da cidade de Uberlândia são de origem exótica (SILVA et al., 2002). A utilização expressiva de espécies exóticas pelos psitacídeos estudados é indicativa do seu comportamento oportunista e da sua capacidade de explorar recursos relativamente novos e incomuns (SIMÃO et al., 1997). Embora plantas exóticas possam afetar negativamente espécies da flora e da fauna (MICHELAN et al., 2010; GLEDITSCH; CARLO, 2014), algumas aves, como os psitacídeos, podem se beneficiar das frutas e sementes dessas plantas. Assim, elas facilitam o estabelecimento de populações de psitacídeos no meio urbano, aumentando a gama de recursos potencialmente exploráveis. 
Brotogeris chiriri foi considerada uma espécie residente e comum em vários estudos realizados em Uberlândia (FRANCHIN; MARÇAL JÚNIOR, 2002; 2004; TORGA et al., 2007; VALADÃO et al., 2006a; 2006b). Além disso, em um estudo realizado no interior do estado de São Paulo, em áreas rurais e campos universitários com diferentes níveis de perturbação antrópica, constatou-se que $B$. chiriri ocorreu preferencialmente nos locais mais alterados (PARANHOS et al., 2007). Portanto, não causa surpresa que, no presente trabalho, esta espécie tenha sido o psitacídeo com maior número de eventos de alimentação. Nota-se ainda que $B$. chiriri apresentou a dieta com o maior número de tipos de itens alimentares, sendo esse um dos fatores possivelmente relacionados com a presença constante dessa espécie nas praças urbanas. Outros estudos têm indicado que espécies de psitacídeos de pequeno porte, tal como $B$. chiriri, podem apresentar ampla plasticidade ecológica, consumindo flores, néctar, polpa, sementes e até mesmo o talo de algumas plantas (ARAMBURÚ; CORBALÁN, 2000; KRISTOCH; MARCONDES-MACHADO, 2001; PARANHOS et al., 2007).

Somente Brotogeris chiriri e Eupsittula aurea apresentaram o comportamento de arrancar e segurar com o pé os itens alimentares. Nos e Camerino (1984) relataram que algumas espécies de psitacídeos têm uma clara preferência para pegar alimentos com o pé esquerdo. Brotogeris chiriri utilizou principalmente o pé esquerdo, o que está de acordo com o encontrado por Paranhos et al. (2007) para essa espécie. Eupsittula aurea, no entanto, utilizou principalmente o pé direito, o contrario do registrado por Paranhos et al. (2009). Psittacara leucophthalmus e D. nobilis, não apresentaram preferência entre os pés, assim como também evidenciado para $A$. pertinax por McNeil et al. (1971). O uso de ambos os pés durante o consumo dos alimentos pode aumentar a diversidade de itens e recursos alimentares na dieta (CANNON, 1982).

A presença de áreas verdes está diretamente relacionada com o aumento da população de aves nos grandes centros urbanos (GUIMARÃES, 2006). Pereira et al. (2005) sugerem que diferentes espécies de psitacídeos se alimentam exclusivamente de frutos oferecidos pela arborização pública. Portanto, o plano diretor de uma cidade deve levar em consideração todas as vantagens que a arborização pública traz para a avifauna nativa (OLIVEIRA, 1990). A diversidade de métodos de aquisição de alimentos apresentados pelos psitacídeos presentes nas praças urbanas da cidade de Uberlândia garante que, embora as áreas de estudo estejam inseridas em um ambiente antrópico, os recursos alimentares disponíveis são comumente explorados. As espécies de psitacídeos registradas utilizaram as praças urbanas para atividades de forrageio, explorando os recursos vegetais encontrados por meio de muitos métodos de obtenção de alimentos, demonstrando uma grande diversidade de hábitos alimentares. Este trabalho também é importante para demonstrar a importância das praças urbanas na manutenção de algumas espécies da fauna, podendo servir como refúgio e fonte de alimento para aquelas que habitam os ambientes antrópicos.

\section{Agradecimentos}

À Julliana Lemes da Costa e Natália Nunes pelo auxílio em campo. Ao prof. Dr. Ivan Schiavani pela ajuda na identificação de algumas espécies vegetais. Ao Dr. Bruno de Souza Barreto pela confecção do Mapa. À Fundação de Amparo à Pesquisa do Estado de Minas Gerais (FAPEMIG) pela concessão da bolsa a CPM, e a Coordenação de Aperfeiçoamento de Pessoal de Nível Superior (CAPES) pela bolsa concedida a VG.

\section{Referências}

ABREU, C. R. M.; VIEIRA, M. F. Os beija-flores e seus recursos florais em um fragmento florestal de Viçosa, sudeste brasileiro. Lundiana, Belo Horizonte, v. 5, n. 2, p. 129-134, 2004.

ARAMBURÚ, R.; CORBALÁN, V. Dieta de pichones de cotorra Myiopsitta monachus monachus (Aves: Psittacidae) en una población silvestre. Ornitologia Neotropical, Montreal, v. 11, n. 3, p. 241-245, 2000.

BAÑOS-VILLALBA, A.; BLANCO, G.; DÍAZ-LUQUE, J. A.; DÉNES, F. V.; HIRALDO, F.; TELLA, J. L. Seed dispersal by macaws shapes the landscape of an Amazonian ecosystem. Scientific Reports, London, v. 7, Article 7373, 2017.

BARROS, R. A. M.; COSTA, C. A.; PASCOTTO, M. C. Diet and feeding behavior of the White-naped Jay, Cyanocorax cyanopogon (Wied, 1821) (Aves, Passeriformes, Corvidae) in a disturbed environment in central Brazil. Brazilian Journal of Biology, São Carlos, v. 74, n. 4, p. 899-905, 2014. 
BENSUSAN, N. Conservação da biodiversidade em áreas protegidas. Rio de Janeiro: Editora da Fundação Getúlio Vargas, 2006. 176 p.

BLANCO, G.; BRAVO, C.; PACIFICO, E. C.; CHAMORRO, D.; SPEZIALE, K. L.; LAMBERTUCCI, S. A.; HIRALDO, F.; TELLA, J. L. Internal seed dispersal by parrots: an overview of a neglected mutualism. PeerJ, London, v. 4, e1688, 2016.

CANNON, C. E. Descriptions of foraging behaviour of eastern and pale-headedrosellas. Bird Behaviour, New York, v. 4, n. 2, p. $63-$ 70, 1982.

CARDILINI, A. P.; WESTON, M. A.; NIMMO, D. G.; DANN, P.; SHERMAN, C. D. H. Surviving in sprawling suburbs: suburban environments represent high quality breeding habitat for a widespread shorebird. Landscape and Urban Planning, Leiden, v. 115, p. 72-80, 2013.

CHITTKA, L.; THOMSON, J. D. Cognitive ecology of pollination: animal behavior and floral evolution. Cambridge: Cambridge University Press, 2004. 360 p.

COLLAR, N. J. Globally threatened parrots: criteria, characteristics and cures. International Zoo Yearbook, Malden, v. 37, n. 1, p. 21$35,2000$.

COLLAR, N. J.; CROSBY, M. J.; STATTERSFIELD, A. J. Birds to watch. The world list of threatened birds. Washington: BirdLife International, 1994. $407 \mathrm{p}$.

COLLAR, N. J.; JUNIPER, A. T. Dimensions and causes of the parrot conservation crisis. In: BEISSINGER, S. R.; SNYDER, N. F. R. (Ed.). New world parrots in crisis: solutions from conservation biology. Washington: Smithsonian Institution Press, 1992. p. 1-24.

DA SILVA, P. A. Seed predation by parakeets Brotogeris chiriri (Psittacidae) in Chorisia speciosa (Bombacaceae). Brazilian Journal of Ornithology, Rio Grande, v. 15, n. 28, p. 3, 2013.

DEL HOYO, J.; ELLIOTT, A.; SARGATAL, J. Handbook of the birds of the world. Vol. 4. Barcelona: Sandgrouse to Cuckoos, Lynx Edicions, 1997. 894 p.

DUGGAN, M. R.; BUCKLEY, D. P.; ANDERSON, M. J. Investigating laterality in the freely occurring behaviours of Budgerigars. South Australian Ornithologist, Adelaide, v. 42, n. 1, 2016.

FONSECA, F. Y.; ANTUNES, A. Z. Frugívoria e predação de sementes por aves no Parque Estadual Alberto Löfgren, São Paulo, SP. Revista do Instituto Florestal, São Paulo, v. 19, n. 2, p. 81-91, 2007.

FORSHAW, J. M. Parrots of the world. Princeton: Princeton University Press, 2010. 404 p.

FRANCHIN, A. G.; MARÇAL JÚNIOR, O. A. Riqueza da avifauna urbana em praças de Uberlândia (MG). Revista Eletrônica Horizonte Científico, Uberlândia, v. 1, n. 1, p. 1-20, 2002.

FRANCHIN, A. G.; MARÇAL JÚNIOR, O. A. Riqueza da avifauna no Parque Municipal do Sabiá, zona urbana de Uberlândia (MG). Biotemas, Florianópolis, v. 17, n. 1, p. 179-202, 2004.

GALETTI, M. Diet of Scaly-headed Parrot (Pionus maximiliani) in a semideciduous forest in southeastern Brazil. Biotropica, Malden, v. 25, n. 4 , p. 419-425, 1993.

GALETTI, M. Seasonal abundance and feeding ecology of parrots and parakeets in a lowland Atlantic forest of Brazil. Revista Brasileira de Ornitologia, São Leopoldo, v. 5, n. 2, p. 115-126, 1997.
GALETTI, M.; GUIMARÃES JR., P. R.; MARSDEN, S. J. Padrões de riqueza, risco de extinção e conservação dos psitacídeos neotropicais. In: GALETTI, M.; PIZO, M. (Ed.). Ecologia e conservação de psitacídeos no Brasil. Belo Horizonte: Melopsittacus Publicações Científicas, 2002. p. 17-26.

GALETTI, M.; RODRIGUES, M. Comparative seed predation on pods by parrots in Brazil. Biotropica, Malden, v. 24, n. 2, p. 222224, 1992.

GLEDITSCH, J. M.; CARLO T. A. Living with aliens: effects of invasive shrub honeysuckles on avian nesting. PLoS One, London, v. 9, e107120, 2014.

GRIMM, N. B.; FAETH, S. H.; GOLUBIEWSKI, N. E.; REDMAN, C. L.; WU, J.; BAI, X.; BRIGGS, J. M. Global change and the ecology of cities. Science, New York, v. 319, p. 756-760, 2008.

GUIMARÃES, M. Há mais aves nos grandes centros urbanos hoje? Ciência \& Cultura, São Paulo, v. 58, n. 2, p. 14-15, 2006.

IBGE - INSTITUTO BRASILEIRO DE GEOGRAFIA E ESTATÍSTICA. IBGE Cidades 2016: Uberlândia, MG. Disponível em: <http://cod.ibge.gov.br/4E6>. Acesso em: 29 agosto 2017.

IDILFITRI, S.; MOHAMAD, N. H. N. Role of Ornamental vegetation for birds' habitats in urban parks: case study FRIM, Malaysia. Procedia - Social and Behavioral Sciences, Amsterdam, v. 68, p. 894-909, 2012.

JANZEN, D. H. Ficus ovalis seed predation by Orange-chinned Parakeet (Brothogeris jugularis) in Costa Rica. Auk, Albuquerque, v. 98, n. 4, p. 841-944, 1981.

KINZIG, A. P.; GROVE, J. M. Urban suburban ecology. In; LEVIN, S. A. (Ed.). Encyclopedia of biodiversity. Vol. 5. San Diego: Academic Press, 2001. p. 733-745.

KRISTOSCH, G. C.; MARCONDES-MACHADO, L. O. Diet and feeding behaviour of the reddish-bellied parakeet (Pyrrhura frontalis) in an Araucaria forest in southeastern Brazil. Ornitologia Neotropical, Montreal, v. 12, p. 215-223, 2001.

LARA, C.; ORNELAS, J. F. Preferential nectar robbing of flowers with long corollas: experimental studies of two hummingbird species visiting three plant species. Oecologia, Heidelberg, v. 128, n. 2 , p. $263-273,2001$.

LORENZI, H. Árvores Brasileiras - Manual de identificação e cultivo de plantas arbóreas nativas do Brasil. Vol. 1. 4 ed. Nova Odessa: Instituto Plantarum, 2002. 384 p.

LORENZI, H.; SOUZA, H. M.; TORRES, M. A. V.; BACHER, L. B. Árvores exóticas no Brasil - Madeireiras, ornamentais e exóticas. Nova Odessa: Instituto Plantarum. 2003. 384 p.

LUCCAS, N. I.; HALLER, E. C. P.; ANTUNES, A. Z. Dieta de psitacídeos (Aves: Psittacidae) no Parque Estadual Alberto Löfgren - SP. Instituto Florestal, Série Registros, São Paulo, v. 40, p. $143-$ 147, 2009.

LUNIAK, M. Synurbization - adaptation of animal wildlife to urban development. In: INTERNATIONAL SYMPOSIUM ON URBAN WILDLIFE CONSERVATION, 4, 2004, Tucson. Anais... Tucson: University of Arizona, 2004. p. 50-55.

MAGAT, M.; BROWN, C. Laterality enhances cognition in Australian parrots. Proceedings of the Royal Society B, London, 2009. DOI: $10.1098 /$ rspb.2009.1397.

MARQUES, R. L. A importância da estrutura do hábitat sobre a composição da avifauna em praças públicas. 2010. $89 \mathrm{f}$. 
Dissertação (Mestrado em Ecologia e Conservação de Recursos Naturais) - Universidade Federal de Uberlândia, Uberlândia. 2010.

McNEIL, R.; RODRIGUEZ, R. J.; FIGUERA, D. M. Handedness in the brown-throated parakeet Aratinga pertinax in relation to skeletal asymmetry. Ibis, Peterborough, v. 113, n. 4, p. 494-499, 1971.

McPHEARSON, T.; PICKETT, S. T. A.; GRIMM, N. B.; NIEMELÄ, J.; ALBERTI, M.; ELMQVIST, T.; WEBER, C.; HAASE, D.; BREUSTE, J.; QURESHI, S. Advancing Urban Ecology Toward a Science of Cities. BioScience, Oxford, v. 66, n. 3, p. 198-212, 2016.

MICHELAN, T. S.; THOMAZ, S. M.; MORMUL, R. P.; CARVALHO, P. Effects of an exotic invasive macrophyte (tropical signalgrass) on native plant community composition, species richness and functional diversity. Freshwater Biology, London, v. 55, n. 6, p. 1315-1326, 2010.

MISSOURI BOTANICAL GARDEN Tropicos: Botanical information system at the Missouri Botanical Garden. 2010. Disponível em: <www.tropicos.org>. Acesso em: 20 mar. 2011.

MOERMOND, T. C.; DENSLOW, J. S. Neotropical avian frugivores: patterns of behavior, morphology, and nutrition, with consequences for fruit selection. Ornithological Monographs, California, v. 36, p. 865-897, 1985.

NOS, R.; CAMERINO, M. Conducta de alimentación de cinco espécies de cotorritas (Aves, Psittacidae). Miscellania Zoologica, Berlin, v. 8, p. 245-252, 1984.

NUNES, A. P.; SANTOS JUNIOR, A. Itens alimentares consumidos por psitacídeos no Pantanal e planaltos do entorno, Mato Grosso do Sul. Atualidades Ornitológicas, Ivaiporã, v. 162, p. 42-50, 2011.

OLIVEIRA, M. M. A. Arborização e avifauna urbana em cidades do interior paulista. Boletim do Centro de Estudos Ornitológicos, São Paulo, v. 7, p. 10-14, 1990.

PARANHOS, S. J.; ARAÚJO, C. B.; MARCONDES-MACHADO, L. O. Comportamento alimentar do Periquito-de-encontro-amarelo (Brotogeris chiriri) no interior do estado de São Paulo, Brasil. Revista Brasileira de Ornitologia, São Leopoldo, v. 15, n. 1, p. 95-101, 2007.

PARANHOS, S. J.; ARAÚJO, C. B.; MARCONDES-MACHADO, L. O. Comportamento de Aratinga aurea (Psittacidae) no sudeste de Minas Gerais, Brasil. Revista Brasileira de Ornitologia, São Leopoldo, v. 17, n. 3-4, p. 187-193, 2009.

PARRINI, R.; RAPOSO, M. A. Associação entre aves e flores de duas espécies de árvores do gênero Erythrina (Fabaceae) na Mata Atlântica do sudeste do Brasil. Iheringia, Porto Alegre, v. 98, n. 1, p. 123-128, 2008.

PEEL, M. C.; FINLAYSON, B. L.; MCMAHON, T. A. Updated world map of the Köppen-Geiger climate classification. Hydrology and Earth System Sciences, Delft, v. 11, n. 5, p. 1633-1644, 2007. PEREIRA, G. A.; MONTEIRO, C. S.; CAMPELO, M. A.; MEDEIROS, C. O uso de espécies vegetais, como instrumento de biodiversidade da avifauna silvestre, na arborização pública: o caso do Recife. Atualidades Ornitológicas, Ivaiporã, v. 125, p. 10-18, 2005.

PIACENTINI, V. Q.; ALEIXO, A.; AGNE, C. E.; MAURÍCIO, G. N.; PACHECO, J. F.; BRAVO, G. A.; BRITO, G. R. R.; NAKA, L. N.; OLMOS, F.; POSSO, S.; SILVEIRA, L. F.; BETINI, G. S.; CARRANO, E.; FRANZ, I.; LEES, A. C.; LIMA, L. M.; PIOLI, D.;
SCHUNCK, F.; AMARAL, F. R.; BENCKE, G. A.; COHN-HAFT, M.; FIGUEIREDO, L. F. A.; STRAUBE, F. C.; CESARI, E. Annoted checklist of the birds of Brazil by the Brazilian Ornithological Records Committee. Revista Brasileira de Ornitologia, Belém, v. 23, p. 91-298, 2015.

PIZO M. A.; SIMÃO I.; GALETTI M. Diet and flock size of sympatric parrots in the atlantic forest of brazil. Ornitologia Neotropical, Montreal, v. 6, p. 87-95, 1995.

PREFEITURA MUNICIPAL DE UBERLÂNDIA. Banco de dados climatológicos de 2011. Uberlândia: Secretaria Municipal de Planejamento Urbano, 2011. 43 p.

RAGUSA-NETTO, J. Exploitation of Erythrina dominguezii Hassl. (Fabaceae) nectar by perching birds in a dry forest in western Brazil. Brazilian Journal of Biology, São Carlos, v. 62, n. 4b, p. 877-883, 2002.

RAGUSA-NETTO, J. Flowers, fruits and the abundance of the Yellow-chevroned Parakeet (Brotogeris chiriri) at a gallery forest in the Southern Pantanal (Brazil). Brazilian Journal of Biology, São Carlos, v. 64, n. 4, p. 867-877, 2004.

RAGUSA-NETTO, J. Extensive consumption of Tabebuia aurea (Manso) Benth \& Hook (Bignoneaceae) nectar by parrots in a tecoma savanna the Southern Pantanal (Brazil). Brazilian Journal of Biology, São Carlos, v. 65, n. 2, p. 339-344, 2005.

RAGUSA-NETTO, J.; FECCHIO, A. Plant food resources and the diet of a parrot community in a gallery forest of the southern Pantanal (Brazil). Brazilian Journal of Biology, São Carlos, v. 66, n. 4, p. 1021-1032, 2006.

RENTON, K. Lilac-crowned Parrot diet and food resource availability: resource tracking by a parrot seed predator. The Condor, Albuquerque, v. 103, n. 1, p. 62-69, 2001.

ROTH, P. Repartião do habitat entre psitacísdeos simpátricos no sul da Amazônia. Acta Amazonica, Manaus, v. 14, p. 175-221, 1984.

SANTOS, A. A.; RAGUSA-NETTO, J. Plant food resources exploited by Blue-and-Yellow Macaws (Ara ararauna, Linnaeus 1758) at an urban area in Central Brazil. Brazilian Journal of Biology, São Carlos, v. 74, n. 2, p. 429-437, 2014.

SECRETARIA MUNICIPAL DE PLANEJAMENTO URBANO. Banco de dados integrados de Uberlândia. Prefeitura Municipal de Uberlândia, MG, 2011. Disponível em: <http:// www.uberlandia.mg.gov.br/uploads/cms_b_arquivos/1428.pdf $>$. Acesso em: 19 dez. 2011.

SHOCHAT, E.; WARREN, P. S.; FAETH, S. H.; MCINTYRE, N. E.; HOPE, D. From patterns to emerging processes in mechanistic urban ecology. Trends in Ecology and Evolution, Cambridge, v. 21, n. 4, p. 186-191, 2006.

SICK, H. Ornitologia brasileira. Rio de Janeiro: Nova Fronteira, 1997. 836 p.

SILVA, E. M.; SILVA, A. M.; MELO, P. H.; BORGES, S. S.; LIMA, S. C. Estudo da arborização urbana do bairro Mansour, na cidade de Uberlândia-MG. Caminhos de Geografia, Uberlândia, v. 3, n. 5, p. 73-83, 2002.

SILVA, G. B. M.; PEDRONI, F. Frugivoria por aves em área de cerrado no município de Uberlândia, Minas Gerais. Revista Árvore, Viçosa, v. 38, n. 3, p. 433-442, 2014.

SILVA, P. A Predação de sementes pelo maracanã-nobre (Diopsittaca nobilis, Psittacidae) em uma planta exótica (Melia 
azedarach, Meliaceae) no oeste do estado de São Paulo, Brasil. Revista Brasileira de Ornitologia, São Leopoldo, v. 13, n. 2, p. 183-185, 2005.

SILVA, T. The golden conure in field and aviary. Avicultural Magazine, Backwell, v. 96, p. 82-88, 1990.

SIMÃO, I.; SANTOS, F. A. M.; PIZO, M. A. Vertical stratification and diet of psittacids in a tropical lowland forest of Brazil. Revista Brasileira de Ornitologia, São Leopoldo, v. 5, n. 2, p. 169-174, 1997.

SNYDER, N.; UGOWAN, P.; GILARDI, J.; GRAJAL, A. Parrots. Status survey and conservation action plan 2000-2004. Cambridge: IUCN, 2000. $180 \mathrm{p}$.

TERBORGH, J.; ROBINSON, S. K.; PARKER, T. A.; MUNN, C. A.; PIERPONT, N. Structure and organization of an Amazonian forest bird community. Ecological Monographs, Ithaca, v. 60, n. 2, p. 213-238, 1990.

TORGA, K.; MARÇAL JÚNIOR, O.; FRANCHIN, A. G. A avifauna em uma seção da área urbana de Uberlândia, MG. Biotemas, Florianópolis, v. 20, n. 1, p. 7-17, 2007.
VALADÃO, R. M.; FRANCHIN, A. G.; MARÇAL JÚNIOR, O. A avifauna no Parque Municipal Victório Siquierolli, zona urbana de Uberlândia (MG). Biotemas, Florianópolis, v. 19, n. 1, p. 77-87, 2006a.

VALADÃO, R. M.; MARÇAL JÚNIOR, O.; FRANCHIN, A. G. A. Avifauna no Parque Municipal Santa Luzia, zona urbana de Uberlândia, Minas Gerais. Bioscence Journal, Uberlândia, v. 22, n. 2, p. 97-108, 2006b.

WRIGHT, T. F.; TOFT, C. A.; ENKERLIN-HOEFLICH, E.; GONZALEZ-ELIZONDO, J.; ALBORNOZ, M.; RODRÍGUEZFERRARO, A.; ROJAS-SUÁREZ, F.; SANZ, V.; TRUJILLO, A.; BEISSINGER, S. R.; BEROVIDES, A. V.; GÁlVEZ, A. X.; BRICE, A. T.; JOYNER, K.; EBERHARD, J.; GILARDI, J.; KOENIG, S. E.; STOLESON, S.; MARTUSCELLI, P.; MEYERS, J. M.; KATHERINE, R.; RODRÍGUEZ, A. M.; SOSA-ASANZA, A. C.; VILELLA, F. J.; WILEY, J. W. Nest poaching in parrots. Conservation Biology, San Francisco, v.15, p. 710-720, 2001. 